\title{
EXPERIMENTS ON THE EMISSION QUANTA OF CHARACTERISTIC X-RAYS.
}

\author{
By David L. Webster.
}

\section{INTRODUCTION.}

T $\mathrm{T}$ has been assumed by many authors that Planck's quantum hypothesis when applied to the energy of cathode rays, would give the highest frequency of $\mathrm{X}$-rays they could produce. The first accurate experimental proof of this law was given by Duane and Hunt ${ }^{1}$ for the case of the general radiation from a tungsten target, and was extended to a cathode ray potential of I Io kv. by Hull. ${ }^{2}$

Off hand one would expect the law to apply to characteristic rays also. The possibility that it does not is indicated by the fact that Whiddington ${ }^{3}$ found that the total radiation from a metal exposed to X-rays begins to increase rapidly with increasing energy of the cathode electrons when their energy is nearly 40 per cent. above that which the quantum law was later found to demand for the production of the $\alpha$ lines, the strongest of the $\mathrm{K}$ series. A similar conclusion on $\mathrm{X}$-rays from a metal exposed directly to cathode rays is indicated by some later work of Beatty. ${ }^{4}$ As the point where this occurs is not very definite and the interpretation of the results is uncertain, it seemed desirable to test each characteristic line alone with a spectrometer. A brief report of the principal results of such a test is contained in a paper which I presented to the American Physical Society in December, 1915, and published in the Proceedings of the National Academy in March, I9I6. The purpose of the present paper is to report the results more fully and discuss in more detail their theoretical significance.

\section{Apparatus.}

For this work I used a rhodium target in a tube which Dr. W. D. Coolidge, of the General Electric Company, very kindly had made up with one of his hot-wire cathodes. The spectrometer worked on the same principle as Bragg's, though the electrical system was somewhat

${ }^{1}$ Amer. Phys. Soc., April, I9I5; Phys. Rev., Aug., I9r 5.

2 Amer. Phys. Soc., Nov., I915; Phys. Rev., Jan., 1916.

8 Roy. Soc. Proc. Ser. A85, July 5, I9II.

4 Roy. Soc., Proc. Ser. A 87, Dec. I3, I912. 
different. The electroscope, instead of being under the axis, was in a brass chamber, placed against the back end of the ionization chamber, whose high potential electrode was a stiff wire passing through an amber plug with a guard ring to the support of the electroscope leaf. The leaf was observed through a microscope mounted on the same arm that carried the ionization chamber. Its sensitiveness was about Io divisions per volt at 60 volts, the potential ordinarily used; the leakage was about I division in 6 minutes, and the drop with a fairly strong reflection was 20 or 30 divisions per second. With reflections of less intensity its readings would repeat themselves with an accuracy of about I per cent.

The crystal face was a very good ( $I, 0,0)$ face of calcite, whose irregularities were so small that they barely showed at all in photographs of the general radiation reflected by the crystal when its position was fixed and the slit was wide enough to include its whole face. The use of such a good surface made it easy to obtain comparisons of intensities in a definite narrow region of the spectrum by using the whole radiation reflected at a fixed position of the crystal with a fixed slit.

To guard against changes of the zero of angle due to heating or cooling of the support of the target, the tube was run continuously throughout each series of readings and the current was made to vary inversely with the potential. The intensities of rays given below are all reduced to a basis of I milliampere in the tube.

The current was furnished by 20,160 Planté cells, capable of giving 42 to $44 \mathrm{kv}$., or within 400 volts above any lower potential for which they were connected. The adjustment within this 400 volts was made by a variable water resistance. The drop in the water and battery together was kept under $\mathrm{x}, 000$ volts, so that small changes in the current could produce only very small changes in the potential on the tube. This potential was measured by an electrostatic voltmeter designed by Dr. Chaffee, consisting of a small dumbbell on a bifilar suspension at ground potential, placed between two larger balls at the high potential and making an angle of $20^{\circ}$ to $30^{\circ}$ with their line of centers. The system was enclosed in a box lined with tinfoil and the deflection of the dumbbell was read with a telescope and scale. This voltmeter was calibrated up to $18 \mathrm{kv}$. by a manganin resistance of 894,200 ohms. Above that point the calibration was done by measuring the voltages of two parts of the battery, first separately, then in series, and then separately again. The errors in this apparatus were probably not over I per cent. Neighboring potentials could be compared, in any one series of measurements, to about o.r per cent. The current through the tube was measured with a milliammeter through which a point in the heating circuit of the 
cathode was connected to the grounded end of the water resistance, the anode being connected directly to the positive pole of the battery.

The apparatus was so arranged that I could watch the milliammeter and voltmeter, and adjust the potential with the water resistance if necessary, from the start to within a few seconds of the finish of each electroscope run. Thus very little error could arise from the slow decline of voltage of the battery.

\section{The Quantum Potentials of the Characteristic Lines.}

If the potential is gradually raised while the spectrometer is set for rays of a frequency not characteristic of the target, no rays will be detected until the potential reaches the value given by the relation

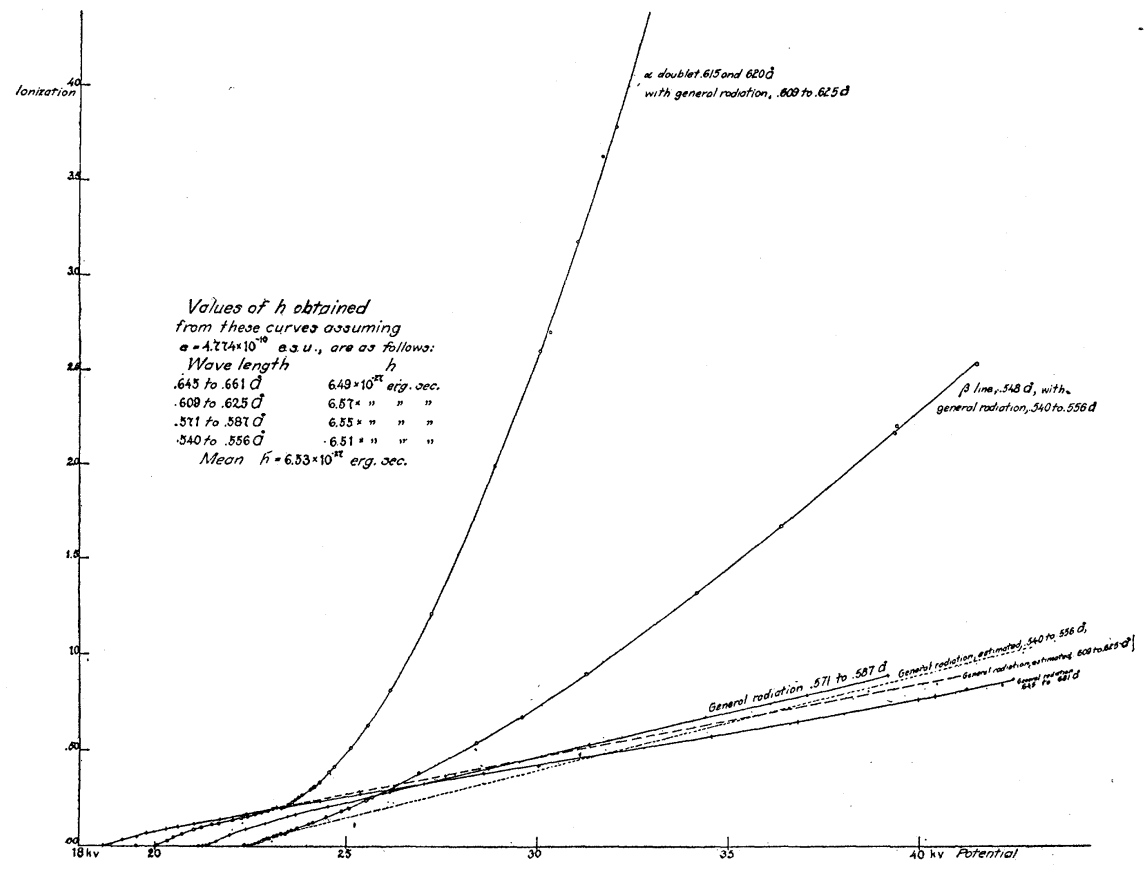

Fig. 1.

$e V=h \nu$ applied to that frequency. At this point the general radiation of that frequency will appear, and its intensity will then increase continuously with the potential. It follows that if the spectrometer is set for a characteristic frequency, it is reasonable to expect, in addition to the characteristic rays, some general radiation obeying Planck's law as at other frequencies. Whether the characteristic rays also obey it is the point to be tested here. 
The results of such a test are shown by the graphs in Fig. I. From these it is evident that the predictions about the general radiation are fulfilled; but the characteristic rays of the $\alpha$ doublet appear, in addition to the general radiation, only above a definite potential about I6 per cent. higher than the quantum value. Above this point, instead of increasing nearly linearly, like the general radiation of that frequency, the characteristic rays increase at a continually accelerated rate. Thus they begin to be a prominent part of the total radiation only at higher potentials. This explains why Whiddington and Beatty did not detect their presence in

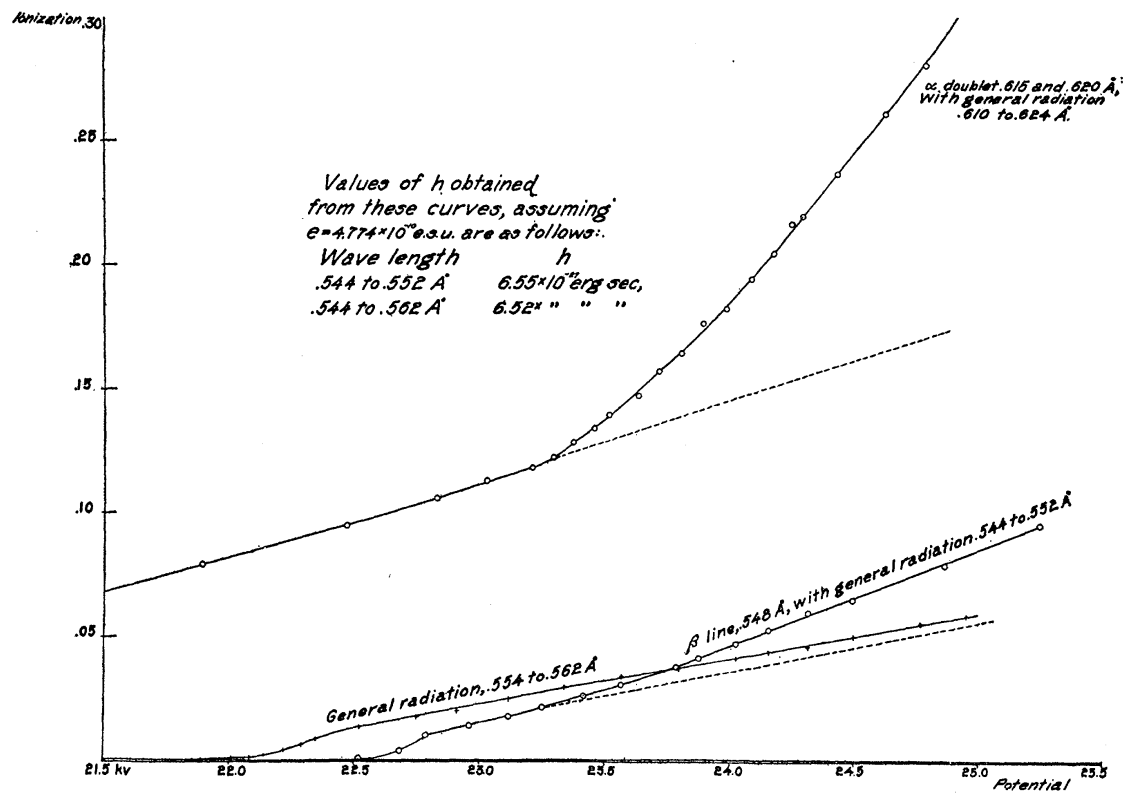

Fig. 2.

the total radiations from other metals at potentials less than 30 to 40 per cent. above the quantum value. The $\beta$ graph indicates similar laws for that line, but not with any certainty when plotted on the 3 cale of Fig. I.

The question now arises, how definite is the point at which the $\alpha$ doublet appears? That is, does the curve shown here have a real discontinuity of curvature or of both slope and curvature, or of neither? One might presume neither, from the rounding of the bottoms of the general radiation curves, but it is easy to show that this is due chiefly, if not entirely, to the width of the slit and the consequent lack of homogeneity of the rays. The slit in this case was wide enough to include 
Physical Review, Vol. ViI., Second Series. June, I9I6.
Plate I.

To face page 603 .

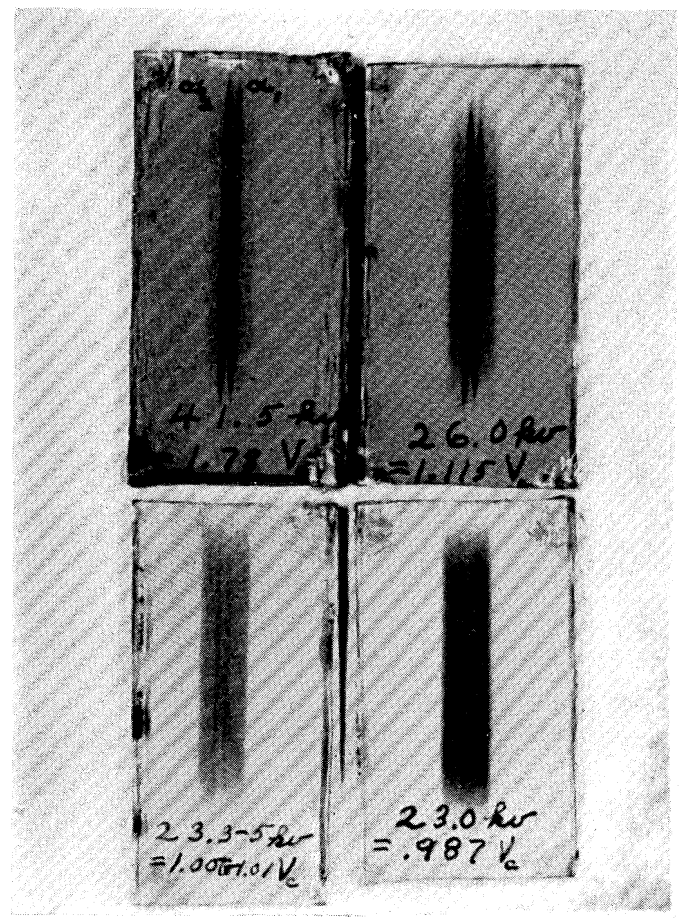

Fig. 3.

These photographs, and those of Figs. 4 and 5 , have been intensified by pasting the gelatine sides of the negatives on a piece of white cardboard and photographing this combination onto a second plate. The contrast is therefore twice as good as that given by the ordinary printing process.

DAVID L. WEBSTER. 
the whole $\alpha$ doublet and a small range of general radiation on each side. This arrangement was adopted as a precaution against shifts of the focus point with potential or temperature. The boundaries of the region used are slightly indefinite, but certainly include no rays beyond the limits given with the graphs.

To obtain more evidence on the sharpness of the corner in this curve, I have taken seven series of measurements on the $\alpha$ doublet under good conditions, and four on the $\beta$ line. One series of each kind, with a similar series for a part of the general spectrum near $\beta$, is reproduced on a larger scale in Fig. 2. If the corners in these curves are really discontinuities in either slope or curvature, it is of course impossible to prove it definitely, and all that can ever be said is that the deviations from curves with such discontinuities are less than a certain amount. With the battery and other apparatus now available such a statement can be made of discontinuities of both curvature and slope in the $\alpha$ curve, with limits of error of I per cent. in intensity and o.I per cent. in potential. The $\beta$ line seems to have similar discontinuities, and apparently the critical potential is the same for both lines. As a matter of fact there are theoretical reasons, discussed below, for doubting whether the discontinuities of slope are real, and in some of the series of measurements there seem to be traces of upward curvature below the critical point, but the change of slope is certainly very sudden. The potential at which the maximum curvature of the $\alpha$ graph occurs is $23.3 \mathrm{kv}$. $\pm \mathrm{I}$ per cent.

To prove more definitely that this is the point at which the lines first become appreciable, I have taken a series of photographs of the $\alpha$ doublet at different potentials, as shown in Fig. 3. These were taken with the crystal fixed and the slit open wide enough to include about .024A of the spectrum. The exposures differed in such a way as to make the background of general radiation show stronger in the low voltage pictures, although really it is less intense; but in spite of this the lines are stronger at $41.5 \mathrm{kv}$. than at 26.0 , barely visible at 23.3-5, and totally invisible at 23.0 . The steadiness of the apparatus is shown by the boundaries of the general radiation, which are as definite as the width of the source allows them to be. A motion of almost any part of the apparatus would blur them, and a motion of the crystal alone would even blur them without blurring the lines. To test the lines at a potential even nearer the critical one, I took the pictures shown in Fig. 4. For these pictures I first took a series of intensity-potential measurements near the critical point, to determine accurately its position on the voltmeter scale, then exposed at a potential 0.997 times the critical value, or only $0.08 \mathrm{kv}$. below it, then immediately repeated the intensity-potential series to be sure everything 
was satisfactory, and then took the high voltage picture to be sure the lines were in the center of the field. The fact that no lines are visible at the lower voltage seems to prove that the critical potential found by the graphs really does mark the first appearance of any appreciable quantity of characteristic rays.

\section{Comparison of Lines above the Critical Potential.}

This point being settled, the question arises whether the characteristic lines, which all appear at the same potential, show any similarity of behavior above it. The photographs in Figs. 3 and 4 show no obvious difference in the behavior of $\alpha_{1}$ and $\alpha_{2}$, and even those of Fig. 5 show no certainty of change in their ratio. Such a test is necessarily very inaccurate, especially as the presence of different amounts of general radiation impairs the accuracy of both the eye and the plate. Nevertheless, when one remembers that the exposure at $39 \mathrm{kv}$. was only 8 minutes and at $23.4 \mathrm{kv}$. I6 hours, or nearly I,000 minutes, and that the current varied inversely as the potential, it appears that the $\alpha_{2}$ line is about 200 times as strong in the former picture as the line and the general radiation back of it in the latter. The lines alone, according to the ionization measurements, must be well over I, ooo times as strong in the former as in the latter. Thus even such a rough test as this acquires some value.

The relation between the $\alpha$ doublet as a whole and the $\beta$ line is obtainable from the graphs in Fig. I, where we may extrapolate for the amount of general radiation with each line as shown in the dotted curves. The points where these cross the other general radiation curves may be obtained approximately from the fact that such a crossing signifies a maximum of intensity in the spectrum about half way between the wavelengths concerned, and that this maximum shifts steadily to the shorter wave-lengths with increasing potential. The curves thus obtained have the right slopes at the critical potential and are confirmed within the limits of error by plotting the spectra at different potentials, as shown below. Subtracting the estimated general radiations from the total intensities, one obtains the curves given in Fig. 6 . The ratio $\alpha / \beta$ is indeterminate at the critical potential and very inaccurate near it, but soon becomes constant. In fact, if the ordinates of the $\alpha$ curve are divided by this constant and plotted, they all lie on the $\beta$ curve, almost within the width of the line used to represent it. The absolute value of this ratio is of little importance, since these "intensities" being ionization currents in an arbitrary amount of ethyl bromide, are on slightly different scales, but the constancy of this ratio seems very significant. 
Physical Review, Vol. Vit., Second Series. June, I9I6.
Plate il.

To face page 604

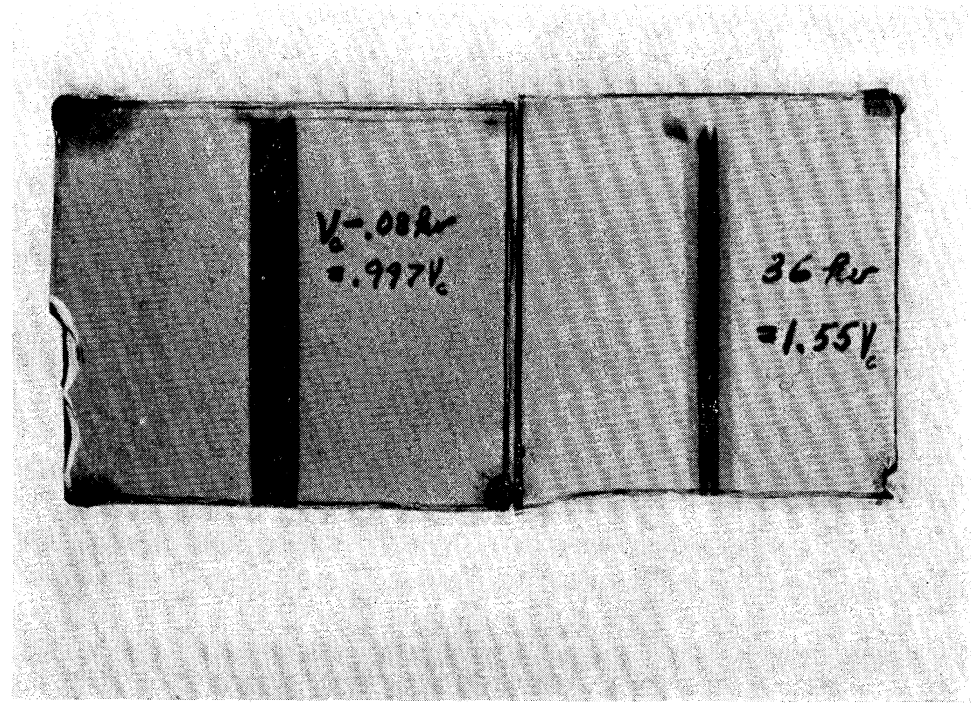

Fig. 4.

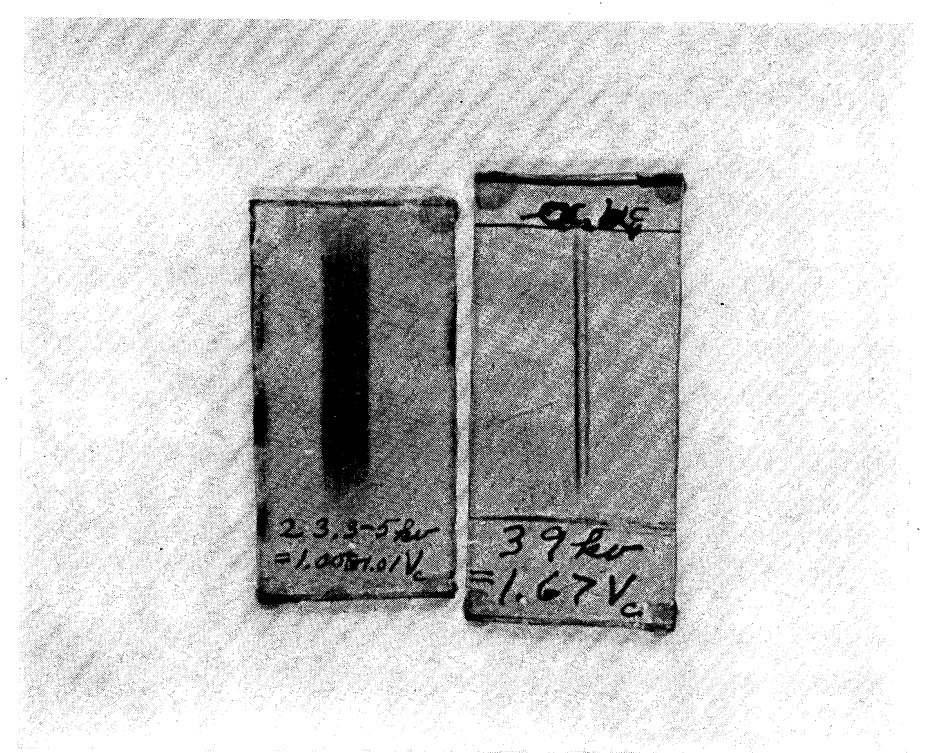

Fig. 5.

Exposure at $39 \mathrm{kv} ., 8$ minutes; at $23.3^{-5} \mathrm{kv}$. , I6 hours.

DAVID L. WEBSTER. 
To test this point by an independent method, I have plotted the spectra shown in Fig. 7. This test is really not so conclusive as the other, because of the long time necessary for determining one of these spectra, and also because of the uncertainty, with the narrow slit used in such measurements, of locating and measuring the exact maximum of each line. The ratios in which the lines increase between $3 \mathrm{I} .8$ and $40.0 \mathrm{kv}$. are nevertheless nearly equal, and the slight inequality is in the direction

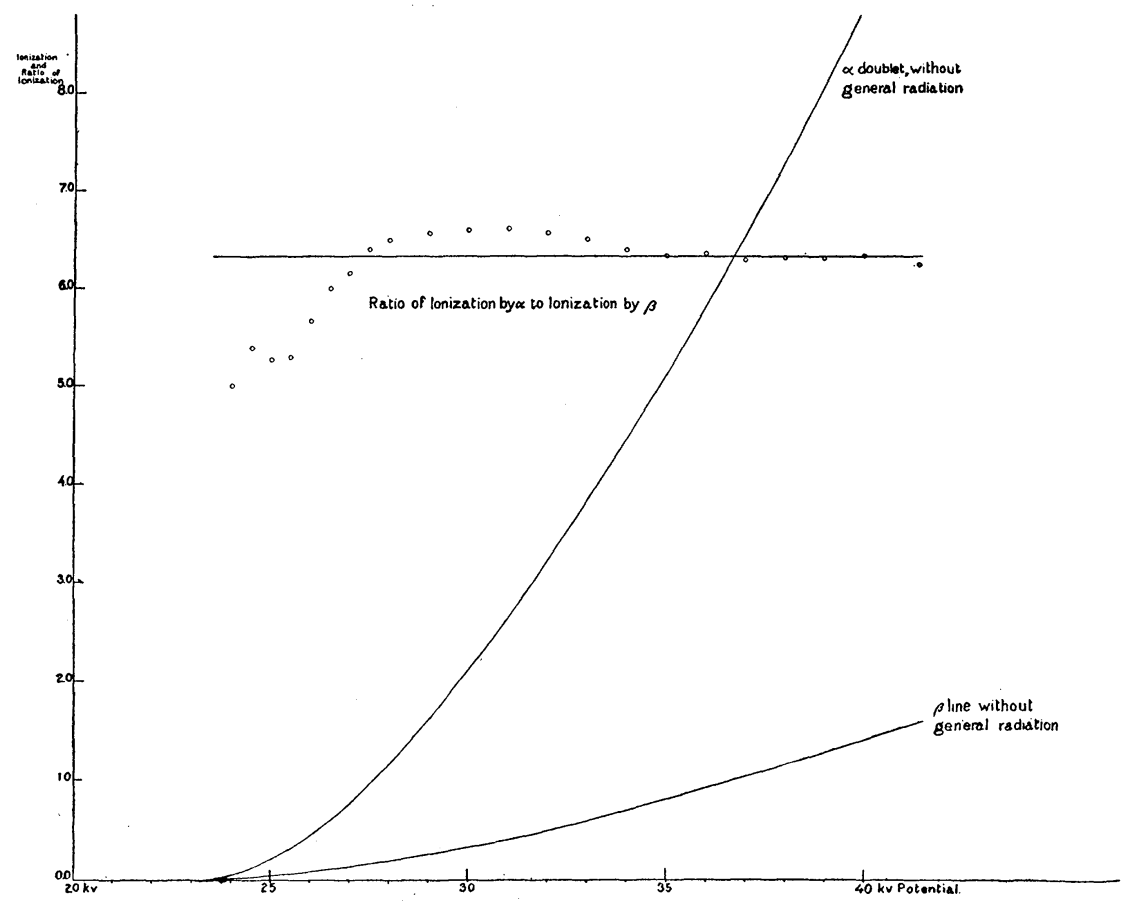

Fig. 6.

opposite to that appearing in the other method. In addition to confirming the previous results these curves show that the $\gamma$ line also increases in the same ratio as the others. For data on the $\alpha_{1}$ line we can take the height of the lower hump in the $\alpha$ curve. The ratio of its intensities seems to be between 2.8 and 3.3, agreeing with the others as well as one can expect of such an uncertain measurement. Hence we may state as a second principle resulting from these experiments that above the critical potential the lines all increase in the same ratio for any given increase in potential. 
The Critical Wave-Length.

These spectra also give some information on other points, such as the way a small impurity of ruthenium in the target produces its lines as if the rhodium were not there, exactly as the zinc showed its presence in a brass target used by Moseley in his well-known series of wave-length measurements.

Another interesting point shown by these spectra is the series of values

Ratios of intensities of lines at $40.0 \mathrm{kr}$. to those at $31.8 \mathrm{kv}$, (with general radiation subtracted), are:

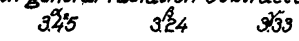
Values. of h given by these curves, assuming e. $4774410^{-10}$ e.s.u., are at $40.0 \mathrm{kv}, \quad h=6.52 \times 10^{-i 2}$ erg sec; " $31.8 \mathrm{n}, \quad h=6.52 \times 10^{-17}$ " "; " $232 ., \quad h=6.59 \times 10^{* \prime} ", "$. Mean of all h determinations on rhodium. with this value of e is $6.59 \times 10^{\text {"th }} \mathrm{erg}$ sec.

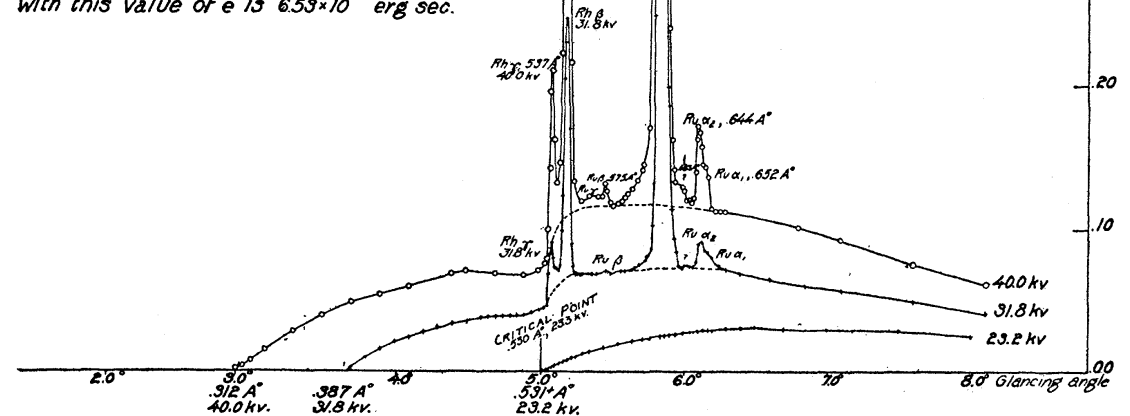

Fig. 7.

of $h$ obtained from their short-wave limits. The agreement between these values is much better than was expected, and they also agree surprisingly well with the mean of the values from the intensity-potential curves.

For all the wave-lengths and $h$ values given here, Bragg's grating space for calcite was recomputed on the basis: $e=4.774 \times 10^{-10}$ esu. (Milli- 
kan), atomic weight of silver $=107.88$, electrochemical equivalent of silver $=.00111827 \mathrm{gm}$. per co., molecular weight of calcite $=$ I00.09, $c=2.9986 \times 10^{10} \mathrm{~cm}$. per sec. (Last four from Kaye and Laby's tables.) These data raise the grating spacing from $3.025 \AA$ to 3.03 , and give for the mean value of $h, 6.53 \times \mathrm{IO}^{-27} \mathrm{erg}$. sec. This lies above the values given by Duane and Hunt ${ }^{1}$ and Planck, ${ }^{2}$ and below those given by Millikan and Hull. ${ }^{3}$

The most important information given by these spectra is the location of the wave-length whose quantum potential is the critical value for the production of the $\alpha$ and $\beta$ lines. For this wave-length, . $530 \AA$, is between I.O and I.5 per cent. short of the $\gamma$ line, and is in just the part of the spectrum where the absorptive power of the rhodium increases suddenly with decreasing wave-length. Some evidence of this increase of absorption is given here by the drop of intensity of the general radiation. For this drop is undoubtedly due to the slight roughening of the surface of the target by the cathode rays, combined with the fact that the rays leave it at a very small angle to the surface and therefore the humps. must screen off the more easily absorbed rays coming from the hollows. In fact Hull ${ }^{4}$ has shown that in the cases of tungsten and molybdenum: the spectrum without such screening shows neither a rise nor a drop near the $\gamma$ line, but with a thin screen of the same material as the target the spectrum is deformed in exactly this way. As a matter of fact I find that the point where this occurs seems to be at the $\gamma$ line itself, rather than the critical wave-length found by potentials, but as I shall point out below, the latter is probably too short. I have also obtained several photographs which show that this change does occur at the $\gamma$ line; but these are not suitable for reproduction, and an accident to the apparatus has rendered further work impossible for the present. ${ }^{5}$

We may now state the results of the present experiment in the form: The $K$ series rays from a rhodium target are emitted in appreciable quantities only if the potential is great enough to excite general radiation of a wave-

${ }^{1}$ Amer. Phys. Soc., April., I915; Phys. Rev., Aug., I9I5. Recomputed on the basis used here, their value is 6.50 .

2 "Heat Radiation," Translation by Masius, p. 172 , gives $6.415 \times 10^{-27}$ erg sec. for $h$ and $4.67 \times 10^{-10}$ esu. for $e$.

${ }^{3}$ Amer. Phys. Soc., Dec., 1915. Their values are 6.57 and 6.59 respectively.

${ }^{4}$ L.c., note 7.

${ }^{5}$ Note added in proofs. Since this was written I have got the apparatus working again, and have obtained a better series of photographs of the $\gamma$ line and its neighborhood, with the tube turned at different angles to the direction of the rays used. These also show a drop of intensity of the general radiation on passing the $\gamma$ line when the angle of the rays from the target is small, but none when it is as large as $10^{\circ}$. This confirms the above statement of the origin of the drop and the location of the discontinuity in the absorption spectrum at the $\gamma$ line itself. 
length slightly less than that of the $\gamma$ line, at which the sudden change of absorptive power occurs. Since the $\mathrm{K}$ series lines in all elements are very similar, the law in this form probably holds for them all. Above this potential the $K$ series lines all increase in the same ratio for any given increase of potential; their rates of increase are continually accelerated, while that of any short region in the general spectrum is at first retarded and then nearly constant. Taking $e$ as $4.774 \times 10^{-10}$ esu, Planck's $h$ appears to be $6.53 \times 1 \mathrm{O}^{-27} \mathrm{erg}$. sec.

\section{The Basis for Interpretation of these Results.}

The significance of these results depends of course on what are the fundamental laws of X-ray phenomena. If these are the laws of electromagnetics as applied to wireless telegraph radiation, we can draw one set of conclusions; if they are laws which would permit the existence of such an atom as Bohr's, our conclusions must be entirely different.

As Hull has pointed out," the critical potential found in these experiments is very consistent with what might be predicted from Kossel's model of the atom, ${ }^{2}$ which contains revolving rings of electrons rather similar to those of Bohr, and determines the frequencies of radiations by the quantum relation applied to the energy of an electron dropping from one ring to another. Such an atom, nevertheless, is known to present many difficulties, such as that of accounting for the stability of the rings in the presence of one another, and even more in the presence of other atoms. Another objection, often overlooked, is that such an atom, even though it does contain the quantum relation, does not satisfy the condition that an oscillator must be able to possess any number of quanta at one time without affecting its frequency. Since this is one of the essential conditions of Planck's development of his law ${ }^{3}$ the failure to satisfy it deprives such an atom of one of the chief advantages of the introduction of the quantum.

Moreover there is the difficulty of accounting for the failure of the rings to lose energy rapidly by radiation, especially now that we have the evidence of X-ray reflection as well as optical phenomena in favor of the electromagnetic wave theory. The argument against the wave theory based on quantum relations does not seem valid, since I have proved in a previous paper ${ }^{4}$ that these relations can be reconciled with

1 Amer. Phys. Soc., Dec., I9r 5.

${ }^{2}$ Verh. d. D. Phys. Ges., Nov. 3o, r9r4; see also Wagner, Ann. der Phys., r9r5, and Cabrera, Nature, Oct. 7 , I9I5.

${ }^{3}$ L. c., p. I53.

4 Amer. Acad. Proc., Jan., I9I5. 
a form of the classical electromagnetic theory containing a few additions suggested by Parson's magneton theory of the atom, ${ }^{1}$ and Planck's method of deriving his radiation law. Consequently the laws of the classical electromagnetics will be taken as a basis for the present discussion. I shall not attempt to give a complete picture of the X-ray mechanism, or even state whether the emitting oscillators are in the nucleus of the atom or outside it, but shall merely attempt to show what conclusions about this mechanism can be drawn from the experiments described above.

\section{The Relation between the General and Characteristic Rays.}

Proceeding along this line, one point may be deduced from the fact that there is a definite high-frequency limit to an X-ray spectrum at a constant potential. By Fourier's series or by direct combination of reflected waves from individual layers of atoms in the crystal, it may readily be shown ${ }^{2}$ that this fact is inconsistent with the pulse theory of $\mathrm{X}$-rays and signifies that $X$-rays of the general spectrum are emitted in periodic trains by oscillators of definite frequencies, even though the spectrum is continuous. Of course there is bound to be a certain amount of irregular, or pulse, radiation, which is impossible to calculate a priori, but which is thus proved to be extremely small.

We can even go a step further and say that the fact that the end of the spectrum can be located within I per cent. proves that there must be at least roo waves in the $\operatorname{train}^{3}$ to give the necessary interference. Probably there are many more.

The existence of oscillators of frequencies distributed throughout the spectrum is indicated also by the evidence of absorption. For the gradual decrease of absorption with increasing frequency, interrupted by a sudden increase near each characteristic series, is not explainable on a basis of oscillators of any one or small number of frequencies, least of all can it be attributed to the characteristic ones. ${ }^{4}$. Consequently it is not surprising that the evidence of the emission spectra also indicates the existence of all frequencies in the target, although not necessarily all in one atom at one time.

1 A. L. Parson, "A Magneton Theory of Atomic Structure," Smithsonian Miscellaneous Collections, Vol. 23, No. II, I9I5.

2 D. L. Webster, Amer. Phys. Soc., April, I9r5; Phys. Rev., July, Igr5.

${ }^{3}$ From a theory of reflection which I published in the Physical Review in March, I9r5, it might appear as if we could find an upper limit to the length of these trains from the depth of penetration of the rays in the crystal. However, other considerations, noted under the heading, "Errata and Addenda," Physical Review, June, rgI6, have shown that the main hypothesis of that theory must be incorrect, and therefore that the effects it was designed to explain must be due to other causes than the shortness of wave trains assumed there.

${ }^{4}$ See $e$. g., Lorentz, Theory of Electrons, p. I54. 
In view of this agreement, it is surprising that where the absorption suddenly increases, near the $\gamma$ line, there is no simultaneous increase of emissivity. ${ }^{1}$ This may be explained by an hypothesis which we shall find extremely useful, namely that the $K$ characteristic rays produced by the action of cathode rays are due to the same mechanism as those produced. by fluorescence.

The natural assumption that they are produced as true fluorescence by absorption of the higher frequency rays entering the target is precluded by the experiments of Beatty ${ }^{2}$ on total radiation. However, when an oscillator does produce the $\mathrm{K}$ fluorescence, it must obtain the energy by absorption from higher frequency X-rays and store it in some non-radiating system such as the magneton theory ${ }^{3}$ provides, until it has acquired a whole quantum. Then it must remit at least a part of this quantum in a complex vibration whose component frequencies are those of the $\mathrm{K}$ lines. The quantum relation would indicate that if its absorbing frequency is not much above that of $\mathrm{K} \gamma$ it probably emits most of its energy in this way. Now if this same mechanism acquires a quantum from a cathode particle, the hypothesis given above indicates that it emits most of this energy likewise as $\mathrm{K}$ radiation. Hence the $\mathrm{K}$ series oscillators will have little effect on the general radiation near the $\mathrm{K}$ lines, and this general radiation must be due to oscillators associated with the L, M, etc., series. Under these circumstances we should expect a lack of any sudden rise of emissivity at the critical frequency of the absorption spectrum, just as it is observed.

The Explanation of the Intensity Relations of the Lines.

The same hypothesis on the origin of the $\mathrm{K}$ rays explains at once why a quantum large enough for one of the higher frequency oscillators is required for the production of any characteristic rays. Likewise it explains the contrast between the upward curvature of their intensitypotential curves and the linearity which I have found to hold for those of the general radiation above this critical frequency as well as below it. For as the potential is raised it increases not only the number of oscillators of a given frequency which a cathode particle can reach and excite, but also the range of frequency of the oscillators for which it has a quantum. Consequently, since each of these factors increases more or less linearly, their product increases as something like the square of either. Thus the intensity-potential curve for the characteristic rays, due to the excitation of such oscillators, should be exactly what is observed.

${ }^{1}$ Cf. Hull. 1. c., note 7.

${ }^{2}$ L. c., note 4.

${ }^{3}$ L. C., notes $\mathrm{I}_{3}$ and $\mathrm{I} 4$. 
Since energy is required for the increased emission of high-speed electrons accompanying the fluorescent $\mathrm{K}$ radiation, it might be supposed necessary to allow for this energy in these comparisons. The most conclusive argument against this supposition is perhaps the work of Barkla and Shearer, ${ }^{1}$ who have found that these electrons have the same energy as those associated with $\mathrm{L}$ radiation. Consequently each electron requires a whole quantum, leaving nothing for secondary $\mathrm{X}$-rays from the atom emitting it. This indicates that the oscillator can emit either an electron or a quantum of X-rays, but not both at the same time. The present discussion is confined to those emitting X-rays only.

If the absorptive power has a finite discontinuity at the critical frequency, as it seems to, it is easy to prove that the present hypothesis leads to finite discontinuities in the curvatures of the characteristic intensity-potential graphs, but none in their slopes. Thus the apparent difference between the wave-length where the absorption changes and that whose quantum potential is the potential of maximum curvature of these graphs is explained as a result of the inability of the apparatus to detect the extremely small amounts of characteristic radiation in the presence of the general at potentials very near the actual critical value. Probably some characteristic rays are produced at any potential above the quantum value for the $\gamma$ frequency, though for the first one or two hundred volts the amount must be very small indeed.

Another experimental fact to be explained is the constancy of the ratio of intensities of any two lines, a state of affairs remarkable for its difference from that of lines of the same series in light. Of course no atom can hold an infinite number of oscillators at one time, as it would have to to produce the whole general spectrum alone. Moreover, the absence of selective absorption of the characteristic rays by the element producing them shows that when the oscillator finishes emitting a set of these rays its frequency must rise again into the region of stronger absorption. Presumably it is a matter of chance what higher frequency it assumes, since any predetermined frequency for an atom would mean a difference between it and other atoms of the same element with different predetermined frequencies. Thus we must conclude that the mechanism emitting characteristic rays is not only the same in the case of direct production as in fluorescence, but also the same for fluorescence from all higher frequencies and direct production with all sufficient potentials. Thus it is evident that we should expect the complex characteristic vibration to be the same and the ratios of its component lines to be the same, in all cases, just as they are found to be.

1 Phil. Mag., Dec., I915. 
Some Evidence on the Mechanism of Absorption.

These data and conclusions are far from being enough for a complete picture of the X-ray oscillator. A mystery which should not be overlooked arises from the fact that if an oscillator has stored up even a large fraction of a quantum from absorbed X-rays it still cannot be made to radiate by a cathode particle having less than a whole quantum, even though the sum of its energy and that of the cathode particle might be almost twice that amount. This fact indeed seems fatal to any model of the oscillator in which the energy absorbed from X-rays is stored as vibratory motion of any particle with which a cathode electron might collide. According to the magneton theory, the stored energy is in the form of a slight increase in the velocity of circulation of the current in the magneton, produced gradually by a mechanism which damps the oscillations on it. There is no obvious way in which a colliding particle could influence this store of energy appreciably, though it might produce a large displacement of the ring and the charge upon it. If this theory is correct, the phenomenon under consideration would indicate that the collision must be elastic unless the energy of these displacements by themselves amounts to a quantum, in which case it may be radiated, rather than returned to the colliding electron.

This assumption is very dubious, being based on but one phenomenon and not as yet subject to test by others. But even if the magneton theory turns out to be incorrect, the facts seem to show clearly an independence of the cathode ray and stored X-ray energies that must have some such counterpart in the modifications they produce in the system.

\section{Summary.}

The chief experimental facts proved here are:

I. Rays of the $\mathrm{K}$ series of rhodium are produced only at potentials above that required to excite general radiation of a frequency equal to or slightly greater than that of the $\gamma$ line.

2. Above this potential all the $K$ lines increase in the same ratio for any given increase of potential; their rates of increase are continually accelerated, while that of any short interval in the general spectrum is at first retarded and then nearly constant.

3. The value of Planck's $h$ appears to be $6.53 \times 10^{-27}$ erg sec. taking $e$ as $4.774 \times 10^{-10} \mathrm{esu}$.

In the discussion of these results it is assumed that $X$-rays must obey the laws of the classical electromagnetics and that the quantum relations must be due to an ability of the oscillator to store absorbed 
energy in some non-radiating system such as the circulation postulated in Parson's magneton theory. On this basis it is shown that:

I. The short-wave limit of the spectrum at a constant potential indicates that even the general radiation consists of wave trains and not of pulses, and therefore that X-rays are emitted by oscillators of definite frequencies distributed throughout the spectrum; this conclusion is strengthened by evidence from the absorption spectrum.

2. All the behavior of the K-rays described above is explainable on an assumption suggested by the general emission and absorption spectra, namely that the same mechanism which emits fluorescent characteristic rays also emits those which arise directly from the impact of cathode rays without true fluorescence.

3. The quantum law for the end of the spectrum indicates that the energy of cathode particle cannot combine with that already stored to produce a quantum; this appears inconsistent with any type of atom in which the energy is stored as vibratory motion, but not necessarily with Parson's theory of the atom.

JefFerson Physical Laboratory, CAmbridge, Mass. 


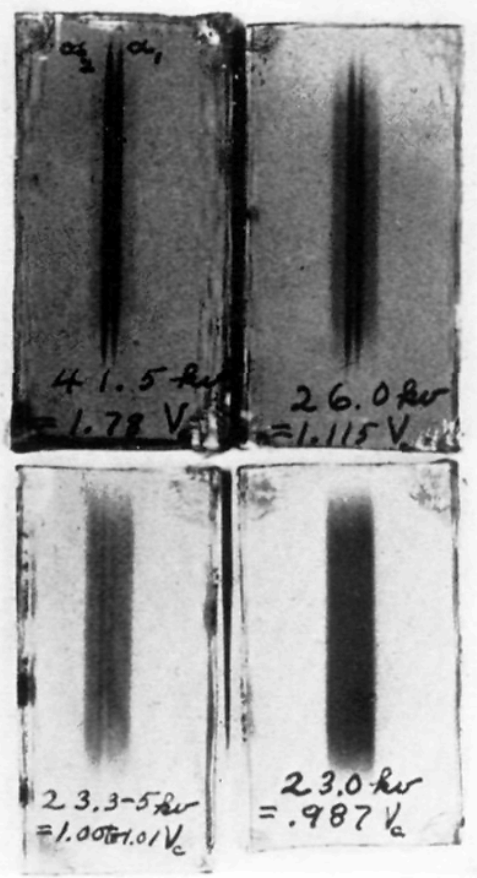

Fig. 3.

These photographs, and those of Figs. 4 and 5 , have been intensified by pasting the gelatine sides of the negatives on a piece of white cardboard and photographing this combination onto a second plate. The contrast is therefore twice as good as that given by the ordinary printing process. 


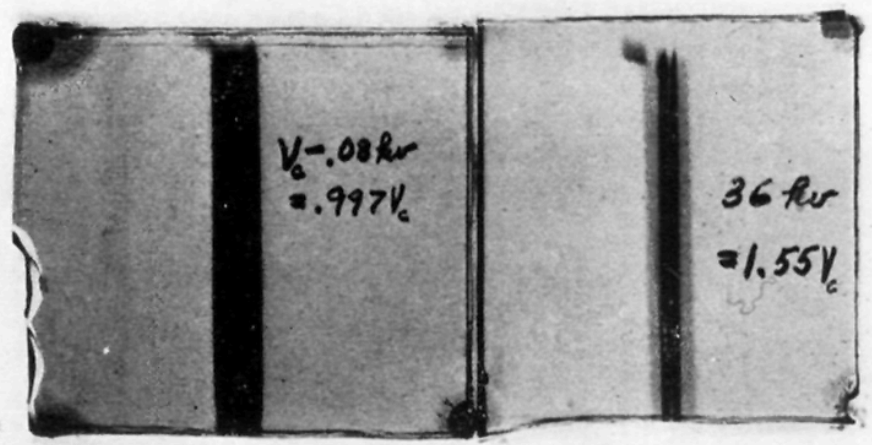

Fig. 4 .

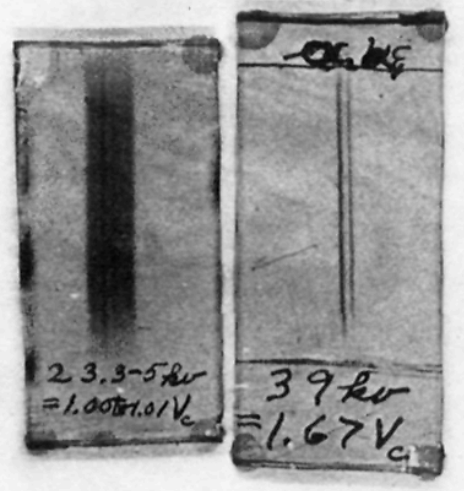

Fig. 5.

Exposure at $39 \mathrm{kv} ., 8$ minutes; at $23.3^{-5} \mathrm{kv}$., I6 hours. 